\title{
вмJ Global Health Systematic review of the concept 'male involvement in maternal health' by natural language processing and descriptive analysis
}

To cite: Galle A, Plaieser G, Van Steenstraeten T, et al. Systematic review of the concept 'male involvement in maternal health' by natural language processing and descriptive analysis. BMJ Global Health 2021;6:e004909. doi:10.1136/ bmjgh-2020-004909

Handling editor Seye Abimbola

- Additional supplemental material is published online only. To view, please visit the journal online (http://dx.doi.org/10. 1136/bmjgh-2020-004909).

Received 30 December 2020 Revised 16 March 2021 Accepted 18 March 2021
Check for updates

(c) Author(s) (or their employer(s)) 2021. Re-use permitted under CC BY-NC. No commercial re-use. See rights and permissions. Published by BMJ.

For numbered affiliations see end of article.

Correspondence to

Dr Anna Galle;

anna.galle@ugent.be

\section{ABSTRACT}

Introduction Experts agree that male involvement in maternal health is a multifaceted concept, but a robust assessment is lacking, hampering interpretation of the literature. This systematic review aims to examine the conceptualisation of male involvement in maternal health globally and review commonly used indicators.

Methods PubMed, Embase, Scopus, Web of Science and CINAHL databases were searched for quantitative literature (between the years 2000 and 2020) containing indicators representing male involvement in maternal health, which was defined as the involvement, participation, engagement or support of men in all activities related to maternal health. Results After full-text review, 282 studies were included in the review. Most studies were conducted in Africa (43\%), followed by North America (23\%), Asia (15\%) and Europe (12\%). Descriptive and text mining analysis showed male involvement has been conceptualised by focusing on two main aspects: psychosocial support and instrumental support for maternal health care utilisation. Differences in measurement and topics were noted according to continent with Africa focusing on HIV prevention, North America and Europe on psychosocial health and stress, and Asia on nutrition. One-third of studies used one single indicator and no common pattern of indicators could be identified. Antenatal care attendance was the most used indicator (40\%), followed by financial support (17\%), presence during childbirth (17\%) and HIV testing (14\%). Majority of studies did not collect data from men directly. Discussion Researchers often focus on a single aspect of male involvement, resulting in a narrow set of indicators. Aspects such as communication, shared decision making and the subjective feeling of support have received little attention. We believe a broader holistic scope can broaden the potential of male involvement programmes and stimulate a gender-transformative approach. Further research is recommended to develop a robust and comprehensive set of indicators for assessing male involvement in maternal health.

\section{BACKGROUND}

Since the 1994 Cairo Conference, where men's involvement in contraception, family

\section{Key questions}

What is already known?

- Increasing male involvement (MI) in maternal health (MH) is considered to be a promising and effective intervention for improving maternal and newborn health outcomes.

- Ml is described as a multifaceted concept in the quantitative literature, although a multidimensional evidence-based set of indicators is lacking.

- In qualitative literature MI is often described by men and women from different settings as the male partner 'being there', meaning giving physical and emotional support.

What are the new findings?

- Conceptualisation of $\mathrm{MI}$ in $\mathrm{MH}$ in the literature is done by focusing on either the psychosocial aspects or on $\mathrm{MH}$ care utilisation. The attention given to one or both aspects resulted in the use of different indicators and depended on the geographical context of the study.

- Overall MI was most often measured by instrumenta actions such as presence at health services, financial support or providing transport. Other aspects of MI, such as communication, emotional support and shared decision making, have received little attention, especially in low-income and middle-income countries.

What do the new findings imply?

- More research into other aspects of Ml (such as the subjective feeling of perceived support and shared decision making) can broaden the potential of MI programmes and also reveal and minimise potential negative side effects of MI interventions.

- A more holistic assessment of $\mathrm{MI}$ in $\mathrm{MH}$, exploring different aspects of $\mathrm{Ml}$, could facilitate researchers to generate more robust findings, strengthening the existing evidence on Ml programmes.

planning, maternal health and child health was emphasised, research has increasingly paid attention to men's role, responsibilities 
and behaviour in sexual and reproductive health. ${ }^{1}$ Evidence about the positive impact of male involvement (MI) in maternal health on maternal and child health outcomes has been widely published in the last decade $\mathrm{e}^{2-6}$ and recently WHO included active involvement of men during pregnancy, child birth and the postpartum period as an effective strategy to improve maternal as well as newborn health outcomes in their 2015 recommendations on maternal and newborn health $(\mathrm{MNH})$ promotion interventions. ${ }^{7}$

However, no common set of evidence-based indicators exists for assessing MI in maternal health, despite considerable evidence about the positive impact. Researchers seem to agree that MI is a multifaceted term but the concept itself has taken different forms according to the context and researcher's interest. Looking through the lens of Prevention of Mother to Child Transmission (PMTCT) programmes, for example, researchers often focus primarily on male presence at antenatal care (ANC) and HIV testing ${ }^{38-10}$ as the core indicators for MI, without paying attention to other aspects of involvement in maternal health. This single measurement assumes that male partner presence is always a positive action and that men who do no not attend services are inherently "not involved'. ${ }^{11}$ However, it is well known that in many health systems men face multiple barriers to being present during ANC such as privacy issues, overcrowded ANC consultations, stigmatisation and strong prevailing gender norms. ${ }^{12-14}$ Consequently, the fact that he is or not present might not correspond to his intentions of being involved or actual (supportive or unsupportive) behaviour outside the health facility. Limited research has also highlighted the negative side of male presence at ANC. ${ }^{12}{ }^{15} 16$ In some cases it might be an act of dominance and control, thereby limiting women's ability to actively participate in the conversation during the ANC consultation. ${ }^{12}$ All these arguments should be taken into account when measuring MI based on a single indicator.

Looking at the qualitative literature there seems to exist some consensus regarding the meaning of MI globally, with slightly different accents according to the context. A study in rural South Africa showed that MI was understood as giving instrumental support to female partners through financial help, helping with physical tasks and providing emotional support. ${ }^{17}$ In Mozambique, MI was seen as 'taking care of the family' in various ways such as providing financial support, making the decisions and showing love towards the partner. ${ }^{12}$ In two Arabic countries, MI was described as being accessible, present and available in addition to being supportive and encouraging. ${ }^{18}$ Studies from the USA found that MI meant 'being there', both emotionally and physically, by doing household chores or listening attentively to the woman's concerns. ${ }^{192}$ African-American parents in the USA summarised MI as being present, accessible, available, understanding, willing to learn about the pregnancy process and eager to provide emotional, physical and financial support. ${ }^{21}$ Despite the common construct of 'being there', often meaning supporting financially, emotionally and being physically present, this has not yet been translated into a set of robust quantitative indicators for measuring MI. Nevertheless more recently studies have started to construct composites or a collection of indicators for measuring MI, instead of focusing on a single item. ${ }^{22}{ }^{23}$ Furthermore, factors such as financial support, birth preparedness, decision making and participation in household chores have been included as MI indicators. Some studies also included reports of the male partner himself, often resulting in contradicting findings between men and women. ${ }^{24}$

Despite the growing number of studies in the field of MI in maternal health, no consensus exists regarding the number and content of indicators for assessing MI in maternal health, although several authors have argued that evidence based indicators are necessary for improving the quality of the available evidence. ${ }^{3} 1625$ With this review, we want to explore to what extent the research community has assessed different dimensions of MI in maternal health and which patterns we can identify in the selection of indicators globally. Only by looking at MI through a broader lens can potential implications of MI interventions on different outcomes such as gender equality, psychosocial health (PSH) and couples birth preparedness be explored and improved.

The primary aim of this systematic review is to examine the conceptualisation of MI in maternal health in the quantitative literature of the last 20 years. As secondary objective we want to critically review and discuss commonly used indicators.

\section{METHODS \\ Protocol and registration}

This systematic review was conducted in accordance with the Preferred Reporting Items for Systematic Reviews and Meta-Analyses statement of 2015 guidelines. The protocol was submitted on the 22 February to International prospective register of systematic reviews (PROSPERO) and published online on the 10 July 2020 under registration number CRD42020169078. Due to the COVID-19 crisis, the PROSPERO register was prioritising submissions related to COVID-19, causing a delay in the registration.

\section{Eligibility criteria}

The systematic review included all types of quantitative studies involving indicators or variables representing MI in maternal health published in the last 20 years. MI was defined as the participation, engagement or support of men in all activities related to maternal health. Maternal health was defined according to WHO as the period from conception until 6 weeks after childbirth, thus covering pregnancy, childbirth and the postpartum period.

A search strategy was developed by AG with inputs from OD. This search was refined with the help of the librarian of Ghent University. The systematic review 
involved a literature search of the PubMed, Embase, Scopus, Web of Science and CINAHL databases for peerreviewed journal articles. Iterative modifications to the original search strategies were conducted, to assure that the observed variations in terminology were adequately reflected in both the index terms and the text-based queries for each database. A final search was conducted on the first of May 2020. Grey literature was identified through the WHO Reproductive Health Library and using Google with relevant keywords. The search strategy for PubMed can be found in online additional file 1 . The outputs of the search were exported to Mendeley desktop V.1.16.1, and duplicates were removed. Subsequently, the titles and abstracts of the studies were imported into Rayyan. Two rounds of screening were applied, first, by title and abstract, followed by full text. Two reviewers independently screened and appraised all eligible articles using preset criteria, and in case of disagreement consensus was reached through discussion. Exclusion criteria were: using only qualitative methodology, systematic review studies, conference abstracts, data collection only in the postpartum period (without any assessment during pregnancy or childbirth), articles without any measurement of the role of the partner, non-English language articles, and studies limited to testing of the male partner for HIV or other sexually transmitted infections without mentioning male support, involvement, engagement or participation. While originally also qualitative studies with a clear conceptualisation of MI were included, it was decided to exclude all qualitative studies for this particular review during the first screening phase because they required a different approach of data extraction and analysis.

In the second stage of screening, full texts were obtained for the screened abstracts. If the article was unavailable through an online search, the article reach system of Ghent University was used to obtain the articles or the authors were contacted to request the full text publication. The same criteria were applied for inclusion and exclusion as in the first stage of screening together with a quality appraisal.

\section{Quality assessment}

Papers selected for retrieval in the second stage were assessed by two independent reviewers for methodological validity prior to inclusion in the review, using standardised critical appraisal instruments from the Joanna Briggs Institute (including the checklist for analytical cross sectional studies, cohort studies, prevalence studies, quasi-experimental studies, randomised controlled trials, case-control studies and systematic reviews). Studies with a score below $50 \%$ were excluded, because they often lacked essential information for this review. A relatively low threshold was used for inclusion because we wanted to examine the concept of MI used by the wider scientific community on a global scale, rather than limiting our results to a few high-quality studies.

\section{Data extraction and analysis}

A pretested data extraction framework in Microsoft Excel was used to extract and chart data from the reviewed articles. The standard data extraction table included authors, publication year, topic of study, the exact term used for describing MI in the study, study design, geographical location of study, definition of MI (if given), indicators used for measuring MI, data sources and quality assessment. Only indicators used in more than five of the included studies were retrieved for the results section and studies referring to a scale of more than 10 items (always psychosocial scales) were categorised as 'psychosocial scale measurement ( $>10$ items)'. Data extraction of every article was done by a team of three researchers. AG screened all articles and GP and TVS independently each screened $50 \%$ of the articles. Disagreement $(<10 \%)$ was resolved by discussion or consultation with one of the supervisors (OD) if needed. Topic allocation was done by an overall thematic analysis of the article, more specifically by reflecting on which particular aspect of maternal health the study was focusing. The topics were inductively created and discussed until agreement was reached among the three reviewers responsible for data extraction. After an initial phase of renaming and discussing the topics, eventually all articles were given one of the following four 'core topics': PMTCT, PSH, abortion and MNH. Articles were categorised as PMTCT if they focused on care for women living with or at risk of HIV (to maintain their health and prevent transmission to their babies), including studies focusing on male HIV testing and prevention. PSH categorised studies focused on social and emotional aspects of male partners' role in maternal health, mainly consisting of articles regarding perinatal depression and stress. Studies categorised as abortion focused on women considering or having experienced an induced or spontaneous abortion. The topic MNH included all studies focusing on MI in MNH, excluding the previous categories (PMTCT, PSH or abortion). The data of the data extraction sheet were cleaned and subsequently analysed using descriptive statistics (more specifically frequencies) and examining associations by inferential statistics. Fisher's exact test was used for assessing differences in the main topic according to terminology and according to continent. In addition, CIs were calculated for visualising differences in proportions in the in use of indicators according to terminology.

For visualisation and confirmatory analysis of the data from the included articles, text mining by $\mathrm{R}$ with the tidytext package was used for natural language processing, ${ }^{26}$ and both the ggplot package in $\mathrm{R}$ and matplotlib package in Python for the graphs. The decision to conduct these analysis was taken after the final screening phase, motivated by the high number of included articles and the nature of the data (including different underlying topics and patterns). First a test set of $20 \%$ of the data was used for writing the text mining scripts, which were refined once the full dataset was entered. The statistic 'term frequency-inverse document frequency' (tf-idf) in 
combination with n-grams was calculated for assessing the importance and structure of certain word combinations within the collection of articles (referred to as 'corpus'). A word's tf-idf represents the frequency of a term adjusted for how rarely it is used. The statistic tf-idf is intended to assess how important a word is to a subset of documents in a collection of documents or corpus. ${ }^{26}$

Lastly, latent Dirichlet allocation (LDA) was used for Topic modelling, which is a commonly used algorithm for topic modelling in text mining, aiming at discovering a given number of topics within a set of documents (=theso-called 'corpus'). ${ }^{26}$ An LDA algorithm automatically generates keywords per topic and their weight (or 'importance'). ${ }^{27}$ Keywords can correspond to more than one topic, but generally with different weights (the keyword will be more or less important in one topic compared with another). This is an advantage of topic modelling as opposed to 'hard clustering' methods: topics used in natural language could have some overlap in terms of words. ${ }^{26}$ An essential step of the LDA algorithm is assigning each word in each article to a topic. As a consequence, each document is composed of multiple topics but typically only one of the topics is dominant. ${ }^{27}$ The more words in a document are assigned to a particular topic, generally, the more weight (also called 'gamma probabilities') will go on that documenttopic classification. ${ }^{26}$ As such, it is possible to determine to which topic every document corresponds dominantly. LDA modelling was used in our study to identify meaningful topics within the complete set of included articles and subsequently allocate every article to one of the generated topics.

\section{RESULTS}

Electronic database searches identified 5277 titles and abstracts, with a further 7 identified through the grey literature search. After removal of duplicates, 3975 articles were screened by title and abstract, resulting in 569 potential articles to be included. After reviewing the full text of these articles, 282 unique studies were included in the systematic review. A flow chart regarding the inclusion of articles can be found as an additional file (online additional file 2).

\section{Characteristics of included studies}

Of all included studies, most studies were conducted in Africa $(43 \%)$, followed by North America (23\%), Asia $(15 \%)$ and Europe $(12 \%)$ (see table 1$)$. The majority of studies collected data from women only $(58 \%)$, while $20 \%$ collected data from both men and women and around $16 \%$ collected data from men only. Registry data were used in $6 \%$ of studies and mostly referred to hospital files indicating the presence of men during ANC. Terms used to assess the role of the male partner were: involvement, support, engagement, participation, attendance and presence. Most studies used a cross-sectional design $(58 \%)$, followed by a longitudinal design (23\%). Only
Table 1 Characteristics of the included studies

n $\%$

\begin{tabular}{|lrr|}
\hline Continent & & \\
Africa & 121 & 42.91 \\
North America & 66 & 23.40 \\
\hline South America & 7 & 2.48 \\
Europe & 35 & 12.41 \\
Asia & 42 & 14.89 \\
Australia & 11 & 3.90 \\
\hline
\end{tabular}

Data source

$\begin{array}{lrr}\text { Registry data } & 16 & 5.67 \\ \text { Survey men } & 45 & 15.96 \\ \text { Survey women } & 163 & 57.80 \\ \text { Survey both } & 55 & 19.50 \\ \text { Others } & 3 & 1.06\end{array}$

Terminology

$\begin{array}{lrr}\text { Involvement } & 140 & 49.64 \\ \text { Engagement } & 6 & 2.13 \\ \text { Participation } & 17 & 6.03 \\ \text { Attendance } & 6 & 2.13 \\ \text { Presence } & 6 & 2.13 \\ \text { Support } & 107 & 37.94\end{array}$

Study design

$\begin{array}{lrr}\text { Cross-sectional } & 164 & 58.16 \\ \text { Cohort/longitudinal } & 64 & 22.70 \\ \text { Quasi experimental } & 20 & 7.09 \\ \text { Experimental } & 16 & 5.67 \\ \text { Case control } & 4 & 1.42 \\ \text { Scale/questionnaire development } & 6 & 2.13\end{array}$

Clearly defined indicator

$\begin{array}{lrr}\text { Yes } & 257 & 91.13 \\ \text { No } & 25 & 8.87\end{array}$

$\begin{array}{lll}\text { No } & 25 & 8.87\end{array}$

Type of indicator

$\begin{array}{crr}\text { Single } & 93 & 34.75 \\ \text { Multiple } & 182 & 63.48 \\ \text { Unclear } & 7 & 1.77 \\ \text { Indicators used } & & \end{array}$

\begin{tabular}{lrr}
\hline Indicators used & & \\
\hline ANC attendance & 113 & 40.07 \\
\hline Financial support or transport & 49 & 17.38 \\
\hline Presence at delivery & 48 & 17.02 \\
\hline HIV testing & 39 & 13.83 \\
$\begin{array}{l}\text { Psychosocial scale measurement } \\
\text { (>10 items) }\end{array}$ & 30 & 10.64 \\
\hline Communication about ANC & 30 & 10.64 \\
\hline Feeling supported & 24 & 8.51 \\
\hline Sharing household tasks & 20 & 7.09 \\
\hline Involvement in decision making & 18 & 6.38 \\
\hline
\end{tabular}

Continued 


\begin{tabular}{|c|c|c|}
\hline & $\mathbf{n}$ & $\%$ \\
\hline Presence at postnatal care & 16 & 5.67 \\
\hline $\begin{array}{l}\text { Involvement in birth preparedness } \\
\text { (not including transport/financial } \\
\text { support) }\end{array}$ & 16 & 5.67 \\
\hline Knowing partner's ANC appointment & 15 & 5.32 \\
\hline Knowledge of danger signs & 12 & 4.26 \\
\hline $\begin{array}{l}\text { Condom use/communication about } \\
\text { condom use }\end{array}$ & 12 & 4.26 \\
\hline Officially registered as father & 9 & 3.19 \\
\hline \multicolumn{3}{|l|}{ Topic } \\
\hline Psychosocial health & 90 & 31.91 \\
\hline $\begin{array}{l}\text { Maternal and newborn health (not } \\
\text { including PMTCT) }\end{array}$ & 134 & 47.52 \\
\hline Abortion & 8 & 2.84 \\
\hline $\begin{array}{l}\text { Prevention mother to child } \\
\text { transmission }\end{array}$ & 50 & 17.73 \\
\hline
\end{tabular}

ANC, antenatal care; PMTCT, prevention of mother to child transmission.

around $6 \%$ of the studies used a randomised controlled trial design. One in 10 studies did not give a clear definition of the indicator used for assessing MI (or one of the similar terms listed earlier). While $35 \%$ of studies used a single indicator for assessing MI, most studies (63\%) used a combination of indicators for measuring MI. Twofifths $(40 \%)$ of studies used ANC attendance as one of their indicators for assessing $\mathrm{MI}$ and one in six studies used financial support or transport $(17 \%)$ and presence during delivery (17\%). Around $14 \%$ of studies used the indicator HIV testing. All indicators that were used to assess MI can be found in table 1. Core topics of the studies were PSH (32\%), MNH (48\%), PMTCT (18\%) and abortion $(3 \%)$. All categories within table 1 were mutually exclusive except for the used indicators.

\section{Terminology used for describing the role of the male partner}

Studies using the term 'male attendance', 'male participation' or 'male engagement' to describe the role of the male partner did not significantly differ from studies using the term 'MI' in the use of the most common indicators (ANC attendance, financial support or transport, presence at delivery, HIV testing, psychosocial scale measurement and communication about ANC). Results of the Fisher's exact test per indicator can be found in online additional file 3 . We consider these terms as synonyms for the remaining results section.

Studies using 'male presence' to describe the role of the male partner always used presence at delivery as their only indicator and differed significantly $(p=0.034)$ in the use of indicators from studies using the term MI.

Studies using the term 'male support' to describe the role of the male partner showed a significant difference $(\mathrm{p}<0.001)$ in the use of indicators compared with the term 'MI'. Studies referring to the role of the partner as 'partner support' used more often complex psychosocial scales ( $>10$ items) such as the Tilburg Pregnancy Distress Scale $^{28}$ or Social Support Effectiveness Questionnaire. ${ }^{29}$

A comparison between the use of indicators for the terms using involvement/engagement/attendance or participation versus support with the respective confidence intervals can be found in figure 1. Studies using the term 'male presence' were excluded from this specific analysis because they always used presence at delivery as single indicator for MI, resulting in 276 included studies $(n=276)$.

\section{The use of different indicators for assessing the role of the male partner}

Among all studies using the term MI/engagement/ attendance or participation, a wide range of indicators was used, often assessed in different ways. We found studies aiming for an in-depth comprehensive assessment of MI through the use of extended surveys for both men and women, combining different indicators $(\geq 3)$

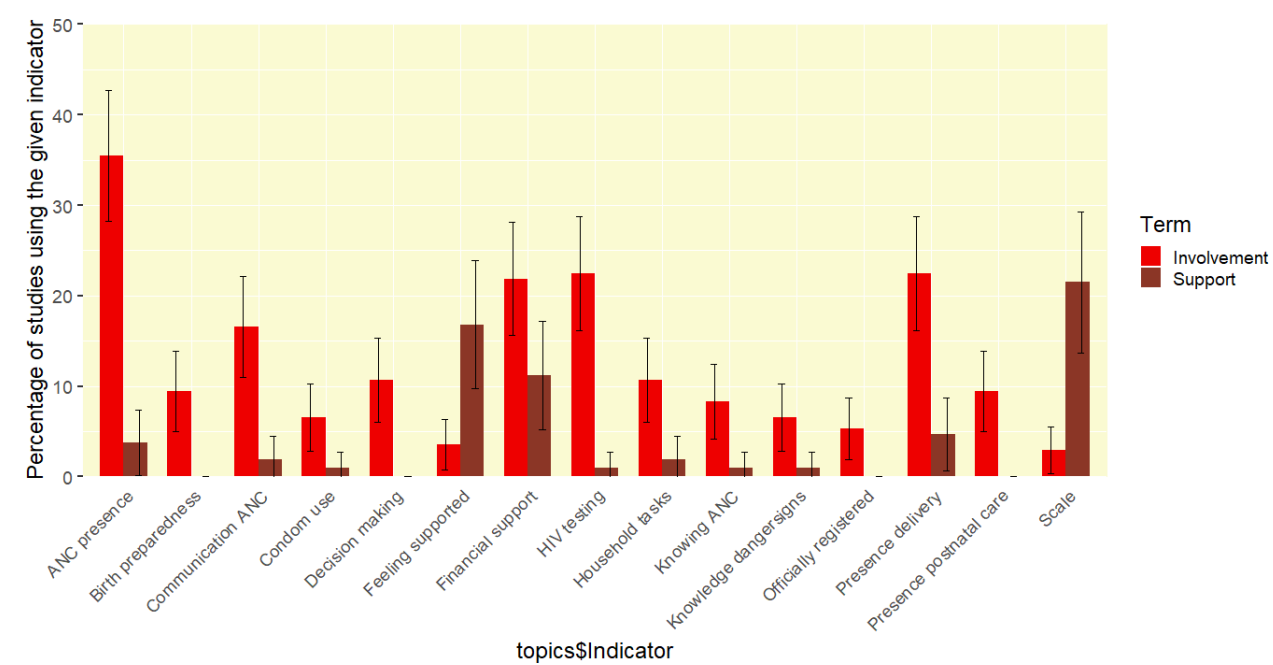

Figure 1 Use of indicators according to the term involvement (engagement/attendance/participation) versus support ( $\mathrm{n}=276$ ). ANC, antenatal care. 
for assessing MI ( $\mathrm{n}=26)$. These studies (see reference list in online additional file 4) were not limited to a certain region or topic, indicating researchers have tried to assess MI in a multidimensional way all over the world. Several authors refer to previously developed scales or indexes, such as the MI index of Byamugisha $e t$ al, ${ }^{23}$ but almost all studies eventually create their own unique composite. We also found studies assessing MI very simply by using service registry data retrospectively and defining $\mathrm{MI}$ as having the father's name written on the birth certificate $(n=7)$. In conclusion, no common set of indicators could be identified for measuring MI/engagement/attendance or participation.

ANC attendance as a single indicator $(n=26)$ was common in studies in low-income countries (LICs), with majority of studies deriving from Africa, Asia and South America and only two studies from North America. On the other hand, presence at delivery was used as an indicator in all continents, suggesting it is a more 'universal' indicator. Financial support, which was used in all continents except in South America, was also used frequently globally as an MI indicator. HIV testing was a typical indicator in African countries and the use of psychosocial scales was more common in North American and European studies.

We were also able to identify some patterns when we examined which studies used less common indicators and why. Studies defining MI by having a father/partner registered on birth certificates $(n=9)$ were most often conducted in North America (six out of nine studies), using big datasets and focusing on neonatal health outcomes. Studies focusing on knowledge of danger signs $(n=12)$ were typically derived from LICs (11 out of 12 studies) and focused on maternal health outcomes (11 out of 12 studies). Studies using condom use (or communication about condom use) as an indicator of MI always derived from an African country and focused in the majority of cases on PMTCT (8 out of 12 studies).

\section{The relationship between terminology, continent of the study and topic}

A scatter plot showing the relationship between the continent of the study, the topic of the study and the terminology can be found figure 2. A significant difference was found in the term used in the study according to continent $(\mathrm{p}<0.001)$ and topic $(\mathrm{p}<0.001)$. Studies using the term 'support' were more often conducted in Europe, North America, Australia and Asia, while the term 'involvement' was most often used in Africa and South America. All continents had studies using both terms.

Looking at the main topic of the study (manually given during data extraction), we also found a significant difference according to continent $(p<0.001)$. The topic 'PMTCT' was most prevalent in Africa while the topic 'PSH' was common for studies from Europe and Australia (see figure 2).

In line with the indicators used for measuring male support (which mostly used psychosocial scales), studies using the term 'support' also more often had as their main topic 'PSH' $(\mathrm{p}<0.001)$ (see figure 2).

\section{Text mining: highly used words according to continent}

Pairs of two consecutive words, referred to as 'bigrams', were examined by text mining using the tidypackage. The tf-idf statistic was calculated for the bigrams in the corpus. Subsequently the top 15 words were ranked per continent. The continents Australia and South America were deleted during the process because of their low number of articles, 11 and 7 respectively. In figure 3, the top 15 words per continent are represented in a word

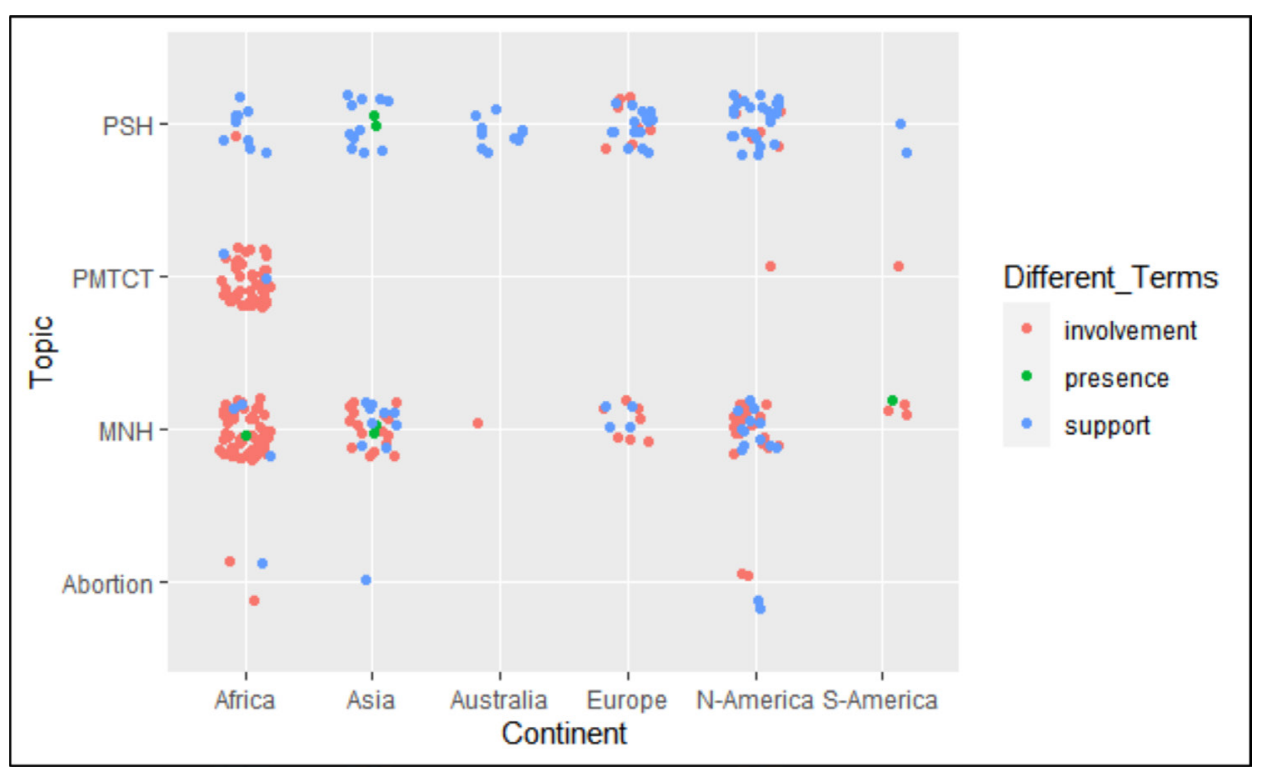

Figure 2 Jittered scatterplot showing the relationship between the manually classified topic, continent and terminology of the different studies included in the review $(\mathrm{n}=282)$. MNH, maternal and newborn health; PMTCT, prevention mother to child transmission; PSH, psychosocial health. 


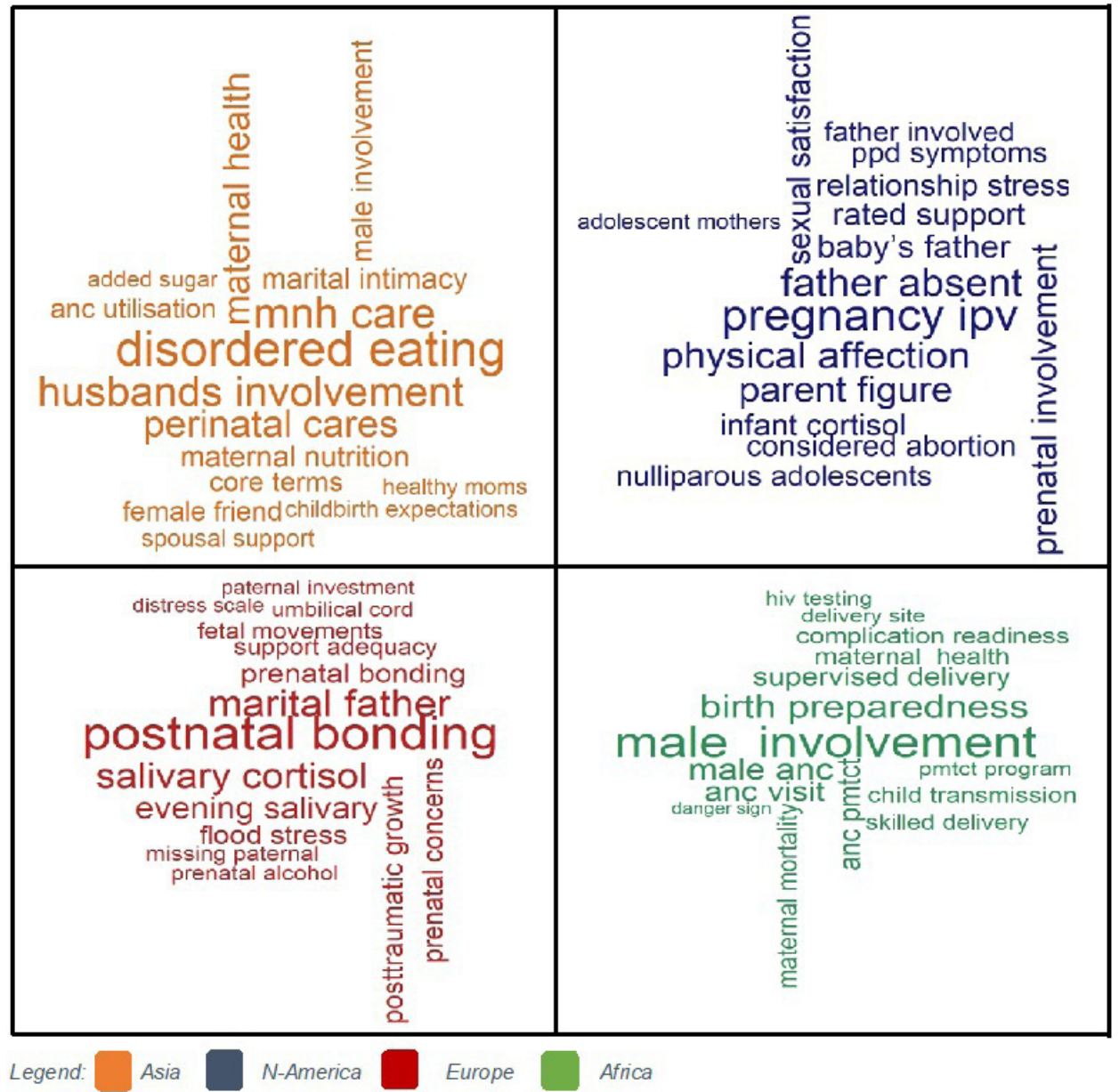

Figure 3 Word cloud visualising the top 15 bigrams per continent, based on their tf-idf value. tf-idf, term frequency-inverse document frequency.

cloud, with font size reflecting the tf-idf value, showing the different content of the articles according to continent. The word cloud shows that in Asia MI studies were characterised by a focus on nutrition (reflected by the words 'maternal nutrition', 'healthy moms', 'disordered eating' and 'added sugar'). In Africa, institutional delivery (reflected by the words 'skilled delivery', 'supervised delivery' and 'delivery site') seem important, as well as PMTCT and birth preparedness. In North America, the words 'relationship stress', 'intimate partner violence' (IPV) and 'postpartum depression' (PPD) show that the literature mainly focuses on PSH and the couple relationship. 'Abortion' and 'adolescents' were more prominent words in the literature in North America compared with other continents.

\section{Latent topic allocation by text mining}

Lastly, we conducted a latent Dirichlet topic allocation by setting $\mathrm{k}=2$, to create a two-topic LDA model. A larger number of topics (ie, a larger $\mathrm{k}$ ) resulted in an unclear pattern of words, which were difficult to interpret. Setting $k=2$ we could find two meaningful topics in the corpus. First the algorithm identified two topics (topic 0 and topic 1) in the corpus and subsequently we calculated the probability that a word corresponded to topic 0 and to topic 1 (the weights) and similarly the probability that a complete document (or article) belonged to topic 0 and topic 1 .

Figure 4 is a grouped bar plot of the top 20 unique key words that are most common within each topic (with the respective word count on the left y-axis and weights on the right y-axis). Especially words with a high weight and low frequency tend to characterise the content of a topic. The top keywords clearly show the two different meanings of the two topics that were extracted from the articles. The most common words in topic 0 include 'HIV', 'PMTCT' and 'test', suggesting that this topic represents the topic of HIV prevention, but also more broadly ANC attendance (by words such as attendance, visit, clinic). The most meaningful words in topic 1 include 'depression', 'stress', 'psychological' and 'anxiety', which suggests it may represent studies around PSH. Furthermore topic 1 includes the word 'father', while topic 0 only includes the word 'male'. This might indicate that studies in the field of PSH have a longer follow-up and more often include the period after birth, when the male partner has become a father. Only unique words were included in the graph for clarity, top key words among both topics included 'women', 'pregnant', 'maternal' and 'child'. 


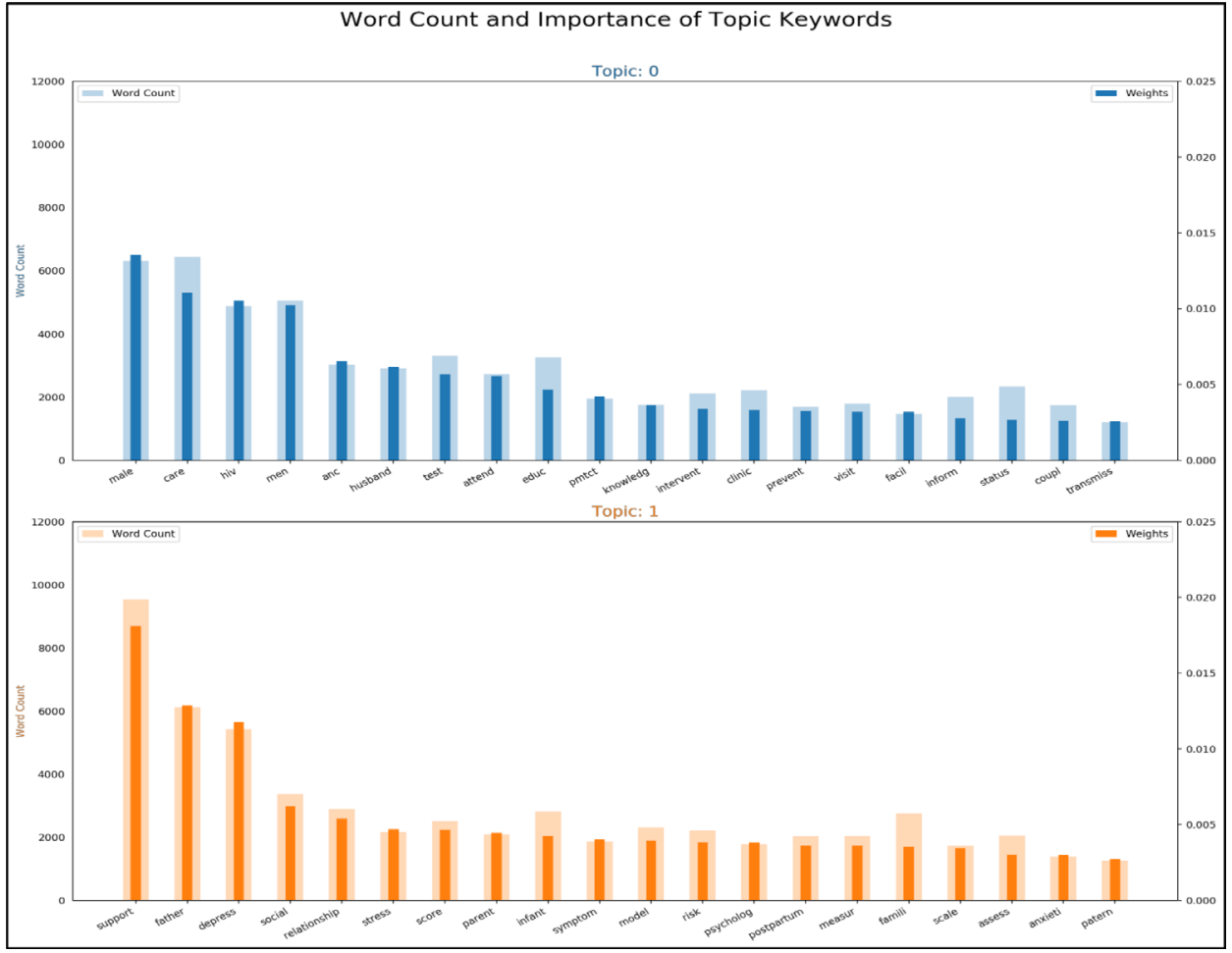

Figure 4 Grouped barplot visualising the top 20 unique keywords per topic with their respective weights and word counts.

The relationship between the computer-driven topic allocation, manually classified topics and continent of the studies

As a confirmatory analysis, we explored how well our unsupervised learning did at distinguishing the different topics in the documents. We would expect that studies that were manually given the topic PMTCT would be found to be mostly (or entirely) part of LDA topic 0 (HIV prevention and ANC attendance) and that studies given the topic 'PSH' would correspond to LDA topic 1 (PSH).
We visualised the relationship between the LDA Topics, manual topics, and continents by a scatter plot (see figure 5) and calculated the associations between LDA topics and manual topics with a cross-tabulation. The latter demonstrated that the topic PMTCT highly corresponds to LDA topic 0 (HIV prevention and ANC attendance), with 92\% (46/50) of the PMTCT studies being classified as LDA topic 0 . The topic PSH strongly corresponded to LDA topic 1 (PSH) with 92\% (83/90) of the PSH studies classified as topic 1. For the topic 'abortion', we saw a higher correspondence with topic $1(75 \% ; 6 / 8)$ ( $\mathrm{PSH})$

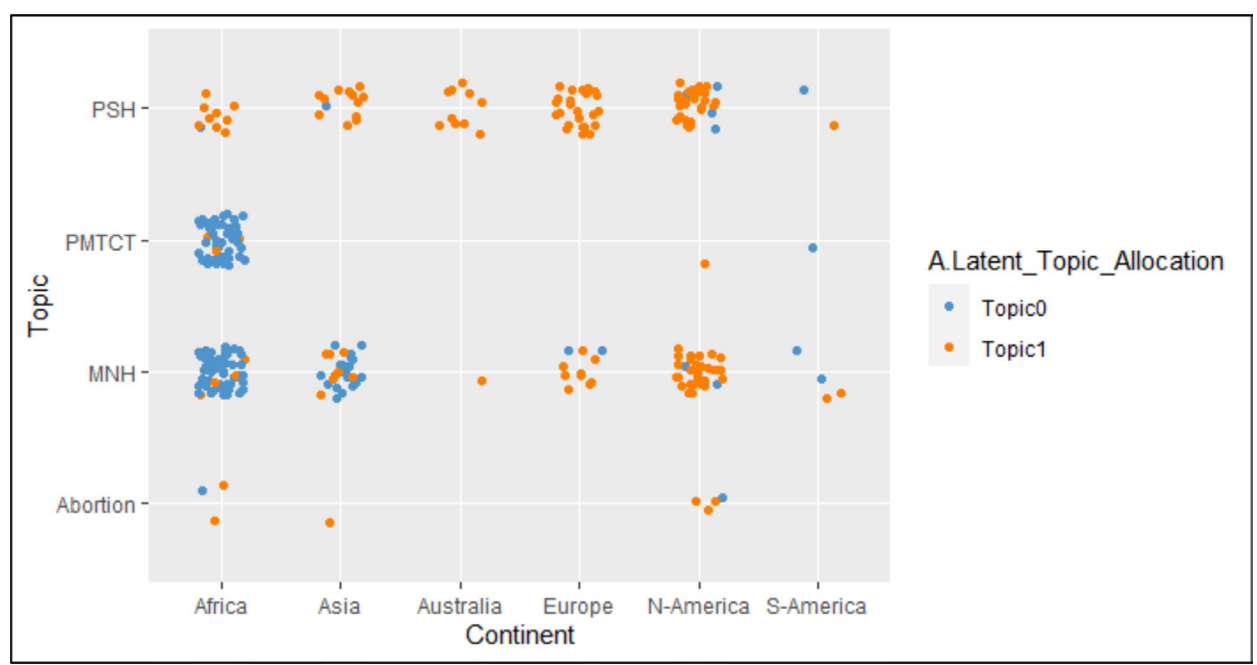

Figure 5 The relationship between the manual topics, LDA topics and continent of the included studies $(n=282)$. LDA, latent Dirichlet allocation; MNH, maternal and newborn health; PMTCT, prevention mother to child transmission; PSH, psychosocial health. 
and lower correspondence with topic $0(25 \% ; 2 / 8)$. The manual topic MNH seemed to correspond to LDA topic $0(61 \% ; 82 / 134)$ and $1(39 \% ; 52 / 134)$. In the scatter plot (see figure 5), we noted a remarkable difference between LDA topic and manual topic correspondence according to continent, whereby $\mathrm{MNH}$ seem to correspond to topic 0 in Africa (HIV prevention and ANC attendance) but to topic 1 in North America (PSH). This indicates that while several studies in North America received the label 'MNH' during our manual data extraction, the natural language of studies in North America clearly differs from those conducted in Africa, resulting in another LDA topic (related to PSH).

\section{DISCUSSION}

The broad range of studies in the scientific literature examining and assessing MI have formed the evidence base for promoting MI as a promising strategy for improving MNH outcomes. ${ }^{2} 3031$ With this systematic review, we aimed to examine the conceptualisation of MI in maternal health in the quantitative literature of the last 20 years and critically review and discuss commonly used indicators. Both manual and computer-driven topic allocation showed us that studies in the field of MI in maternal health are mostly conducted to examine PSH on the one hand and maternal health care utilisation (especially ANC attendance, PMTCT services and institutional childbirth) on the other hand.

Despite the consensus that MI is a multifaceted concept, majority of studies seem to focus on only one particular aspect of the concept, resulting in a simplified measurement of MI in maternal health. The latter was illustrated by the high number of studies relying on a single indicator. Furthermore, there was no common set of indicators among studies using a combination of different indicators, almost every study had its own unique composite. Obviously, the measurement of MI depends on the context, but a critical reflection of the measurement is needed for a correct interpretation of the results. This is especially important as the lack of agreement in indicators leads to the risk that researchers only report the most significant variable. In some studies, included in the review, we found that MI was described and defined as a multidimensional concept in the introduction and methods but that in the results section only one indicator was used as 'the MI indicator'. As a consequence, results might be biased by selecting and reporting only the most significant indicator.

Almost half of the studies focused on presence at ANC or HIV testing and consequently the benefits for mothers and their newborns will mainly be oriented towards the prevention of HIV transmission. This coincides with the implementation of instrumental MI policies in several countries, aiming at improving male attendance at ANC by refusing to attend women without a partner present or giving priority to couples in the waiting line. ${ }^{12153233}$ The negative side effects of introducing such policies for improving male attendance at ANC have started to emerge (such as increased gender inequality, stigmatisation of single women and lower ANC attendance of women ${ }^{1234}$ ), nevertheless they have not led to the elimination of such programmes. This might be related to the strong influence of HIV programmes and donors, where programme success is defined by the proportion of men being tested during pregnancy. ${ }^{35}$ In many communities, men attending antenatal healthcare services are perceived as being HIV positive,${ }^{36}$ because historically HIV counselling and testing was the main reason for inviting men in several African countries. ${ }^{35}$ Future MI programmes should try to shift away from the focus on HIV testing and break the circle of stigmatisation that has been associated with these programmes.

Certain aspects of MI such as communication, decision making and 'feeling supported' were rarely included as MI indicators $(10.64 \%, 8.51 \%$ and $6.38 \%$, respectively) in the studies included in our review, while both quantitative and qualitative research have shown that these aspects of men's involvement play an important role in maternal health care access, utilisation and outcomes. ${ }^{37-42}$ The narrow focus on specific actions of men (such as financial support and ANC attendance) without taking into other aspects (such as couple dynamics and gender equality) clearly entails a risk of missing essential information and underreporting negative consequences. The need for greater incorporation of gender-transformative conceptual approaches into MI interventions, with effective measures, was already emphasised by Comrie-Thomson et al. ${ }^{13}$ Another recent systematic review reported that worldwide only a minority of the interventions aiming at engaging men and boys in sexual reproductive health and rights (SRHR) includes a gender transformative approach. ${ }^{43}$ The authors warn that engaging men and boys in SRHR without explicit attention to gender inequalities can be harmful, particularly when it comes to undermining women's rights and autonomy. Within the field of MI in maternal health, a number of studies has shown that interventions could unintentionally lead to increased domination of decision-making about pregnancy, nutrition and infant care by men, putting pressure on women to adopt certain beliefs and practices. ${ }^{4} 4445$ Also subtle negative effects of MI programmes should be considered, in some cases male presence at ANC might negatively affect women's ability to speak openly and disclose sensitive issues such as IPV. ${ }^{46}$ Only by aiming for a comprehensive assessment of MI programmes (collecting both quantitative and qualitative data) can these issues be identified and addressed in future MI interventions. More data regarding empowerment of women, gender equality and perceived support (from both men and women) can contribute to designing effective interventions with a gender transformative approach. ${ }^{13} 48$ Especially investments in programmes that promote gender equality at an early age (among children, adolescents and young adults) can lead to a 
lifetime of improved health and well-being, ${ }^{49}$ including better maternal health outcomes.

Another interesting finding in our review was that the benefits of MI for the father himself are hardly explored and almost never assessed in interventions. Most studies did not collect data directly from men and even fewer studies assessed the potential benefits of MI for the father himself or mutual perceived support. Furthermore very little is known about men's specific needs during the transition to parenthood. ${ }^{50}{ }^{51}$ Father involvement is almost always used as an instrumental approach to improve maternal health, although the added value for the father himself (eg, the perceived health benefits by improving his own access to healthcare services) were already highlighted during the Cairo conference in $1995 .^{52}$ Emphasising the positive effect for the father himself and investing in his specific needs during the transition to parenthood could be explored as an intervention strategy, ${ }^{5354}$ whereby the health benefits might go beyond his participation in maternal health care services.

Looking at geographical context, several differences were noted by focusing on the most unique and common terms using text mining. In studies deriving from Asia words related to nutrition were more important, while in studies from North America words referring to IPV were more typical. In studies conducted in Europe, stress and depression were important terms, while in Africa ANC attendance and HIV prevention were important. Some of these differences can be explained by the different prevalence of certain problems (such as malnutrition in India ${ }^{55}$ and HIV in Africa ${ }^{56}$ ), while other differences are less logical and probably influenced by funding bias and geographical sociocultural factors.

The proportion of studies about PSH and depression was lower in Africa compared with other continents. However, the literature indicates that perinatal depression is common in the African region. ${ }^{57-60}$ Globally, perinatal depression is estimated to affect around $11 \%$ of women, and recent studies have shown that perinatal mental disorders are at least as prevalent in Africa as in other regions. ${ }^{61-64}$ Furthermore, research has demonstrated that HIV positive women have increased risk of perinatal depression. A systematic review found a prevalence of $23.4 \%$ for antenatal depression and $22.5 \%$ for postnatal depression in HIV infected women. ${ }^{6566}$ The low number of studies on PSH in Africa in our review showed that the relationship between perinatal depression/ PSH and the role of the male partner is poorly studied in Africa. While some have argued that many LICs have more pressing issues within maternal health than addressing perinatal depression (such as severe maternal morbidity and mortality), ${ }^{2}$ other studies have shown there is reasonable evidence for the benefits and effectiveness of psychological interventions in low-income and middleincome countries (LMICs). ${ }^{64} 67$ The low availability of mental health services in LMICs is one of the main challenges for addressing mental health problems, but some recent studies have shown that training and organising lay mental healthcare workers to address mental healthcare problems are a feasible and effective approach to combatting mental health disorders. ${ }^{68}$ Furthermore MI and/or partner support has been shown to be a protective factor against perinatal depression globally. ${ }^{6}{ }^{69-71}$ More research into the field of MI and maternal mental health in LMICs could provide multiple health gains for the male partner, mother and child.

A very low number of studies in our review derived from South America compared with Africa, indicating MI in maternal health care seem understudied in that region. However, the limited literature emphasises that the current level of MI is extremely low in South America, ${ }^{72} 73$ with strong gender norms being the most persistent barrier. ${ }^{74-77}$ The reason why until now very few scientific studies have focused on MI in South America might be related to the lower prevalence of HIV on the continent compared with Africa. ${ }^{56}$ Historically many MI programmes in maternal health in LMIC were implemented in order to improve the uptake of PMTCT, making it less useful (and less funded) in countries with low HIV prevalence. This also explains why the scientific literature on MI in South America is more often focused on attaining gender equality, instead of getting men to health facilities for HIV testing. ${ }^{75} 78$

Lastly but importantly, our review demonstrated that very few studies $(n=8)$ focused on the role of the partner during abortion care. A recent systematic regarding gender transformative interventions for engaging men and boys in SRHR reported a similar gap in the evidence, with very little interventions focusing on engaging men in access to safe abortion care. ${ }^{43}$ However, the important role of the partner in decision making and access to abortion services cannot be ignored. ${ }^{79} 80$ A systematic review from 2016 showed that women contemplating abortion frequently involve their male partner in the decision and rely on him to help with logistics, finances and emotional support before and after the abortion; furthermore, MI was positively associated with women's well-being. ${ }^{80}$ Despite his important role, young men's experiences of unintended pregnancy and their pregnancy decision making are hardly studied within the scientific literature, ${ }^{81}$ although essential for offering adequate counselling and services for men and women regarding sexual and reproductive health. Given that 121 million unintended pregnancies occur each year with $61 \%$ ending in an abortion, more research regarding the role of the male partner in abortion care and pregnancy decisions is highly needed for improving not only maternal health, but also broader SRHR outcomes. ${ }^{43} 7981$ In conclusion, we believe the evidence base on MI in maternal health, and its related indicators, needs to be improved in the future in terms of regional representation, study robustness and a broader holistic scope.

\section{Limitations}

This review has certain limitations. We only included quantitative studies and used qualitative literature only 
for interpretation of the results. By focusing only on quantitative literature and selecting the 'hard core indicators' we, as researchers, also conduct a reductionist analysis of MI, ignoring that certain instrumental actions might mean a lot for women and their partners in terms of involvement and support. A similar in-depth systematic review regarding the qualitative meaning of MI, comparing findings from different regions, would complement our findings. Furthermore, a broader review also including the role of the partner in planning a pregnancy (before conception) and/or family planning decisions could strengthen the existing evidence regarding MI in reproductive health.

Another bias in this study might be related to the principal investigator's (AG) background. A researcher's background and position often affects what they choose to investigate, the angle of investigation, the methods judged most adequate for this purpose, the findings considered most appropriate, and the framing and communication of conclusions. ${ }^{82}$ AG has mainly conducted research in Mozambique, which might lead to a higher interest in the findings most relevant to this context (eg, the relationship between HIV programmes and MI). However, by involving coauthors in all stages of the research process we tried to minimise this bias. Finally, we restricted our search to a selected number of databases and only included English literature, which means certain studies will have been missed.

\section{CONCLUSION}

The concept of MI in maternal health is considered to be multifaceted within the literature but the assessment of the concept differs globally. We found two main streams of conceptualisation within the literature: a focus on psychosocial support on the on hand and focus on instrumental support for maternal health care utilisation (such as PMTCT services, ANC attendance and institutional childbirth) on the other hand. While both aspects are considered as core elements of male partner's (potential) role in maternal health, majority of studies seem to focus on only one of both aspects. In line with these findings the concept of in maternal health it is often measured by a simplified and narrow set of indicators and several essential elements such as communication between the couple regarding maternal health care issues, shared decision making, participation in household tasks and the subjective feeling of being supported by the male partner have received little attention. Until now, very few MI programmes seem to incorporate a gendertransformative approach with adequate measures. In addition, our review identified a gap in the literature regarding the role of the male partner in abortion decisions and access to abortion services. Further research, involving experts and pilot testing, is recommended to develop a robust set of valid and feasible indicators for assessing MI in maternal health globally in a more comprehensive way.

\section{Author affiliations}

${ }^{1}$ ICRH, Department of Public Health and Primary Care, Ghent University, Gent, Belgium

${ }^{2}$ International Centre for Reproductive Health - Mozambique (ICRHM), Maputo, Mozambique

${ }^{3}$ Departamento de Obstetrícia e Ginecologia, Universidade Eduardo Mondlane, Maputo, Mozambique

${ }^{4}$ Department of Human Structure and Repair, Ghent University, Gent, Belgium

Acknowledgements We would like to thank the librarian of Ghent University, Miss Nele Pauwels, for organising training on conducting a systematic review and further personal assistance in developing the search strategy. Furthermore, we would like to thank the colleagues of ICRH-Belgium for their continuous support and encouragement during the study.

Contributors AG conceptualised the study and lead the process of reviewing the literature. GP and TVS screened articles and contributed to data extraction. AG conducted all analysis under supervision of $\mathrm{OD}$. All authors contributed to the development of the manuscript and read and approved the final version. The corresponding author attests that all listed authors meet authorship criteria and that no others meeting the criteria have been omitted.

Funding $A G$ is funded by a VLADOC PhD scholarship from the Flemish InterUniversity Council (VLIR-UOS Belgium).

Disclaimer The funder had no role in the study design, data collection, analysis, interpretation of data or in writing the manuscript. Researchers are independent from funders and all authors had full access to all data (including statistical reports and tables) in the study and can take responsibility for the integrity of the data and the accuracy of the data analysis.

Competing interests None declared.

Patient consent for publication Not required.

Provenance and peer review Not commissioned; externally peer reviewed.

Data availability statement Data are available on request. Data analysed during the current study will be made available from the corresponding author on reasonable request.

Supplemental material This content has been supplied by the author(s). It has not been vetted by BMJ Publishing Group Limited (BMJ) and may not have been peer-reviewed. Any opinions or recommendations discussed are solely those of the author(s) and are not endorsed by BMJ. BMJ disclaims all liability and responsibility arising from any reliance placed on the content. Where the content includes any translated material, BMJ does not warrant the accuracy and reliability of the translations (including but not limited to local regulations, clinical guidelines, terminology, drug names and drug dosages), and is not responsible for any error and/or omissions arising from translation and adaptation or otherwise.

Open access This is an open access article distributed in accordance with the Creative Commons Attribution Non Commercial (CC BY-NC 4.0) license, which permits others to distribute, remix, adapt, build upon this work non-commercially, and license their derivative works on different terms, provided the original work is properly cited, appropriate credit is given, any changes made indicated, and the use is non-commercial. See: http://creativecommons.org/licenses/by-nc/4.0/.

\section{ORCID iD}

Anna Galle http://orcid.org/0000-0003-1427-5067

\section{REFERENCES}

1 Lazarus JV. The changing role of men since ICPD. Entre Nous Cph Den 1999:4.

2 Yargawa J, Leonardi-Bee J, JL-B. Male involvement and maternal health outcomes: systematic review and meta-analysis. J Epidemiol Community Health 2015;69:604-12.

3 Morfaw F, Mbuagbaw L, Thabane L, et al. Male involvement in prevention programs of mother to child transmission of HIV: a systematic review to identify barriers and facilitators. Syst Rev 2013;2:1.

4 Tokhi M, Comrie-Thomson L, Davis J, et al. Involving men to improve maternal and newborn health: a systematic review of the effectiveness of interventions. PLoS One 2018;13:e0191620.

5 Sherr L, Croome N. Involving fathers in prevention of mother to child transmission initiatives--what the evidence suggests. J Int AIDS Soc 2012;15(Suppl 2):17378. 
6 Pilkington P, Milne L, Cairns K, et al. Enhancing reciprocal partner support to prevent perinatal depression and anxiety: a Delphi consensus study. BMC Psychiatry 2016;16:23.

7 WHO recommendations on health promotion interventions for maternal and newborn health [Internet], 2015. Available: https:// apps.who.int/iris/bitstream/handle/10665/172427/9789241508742_ report eng.pdf;jsessionid=62AAA1EB03A463F814C8F3F9 636EFF71? sequence $=1$

8 Theuring S, Mbezi P, Luvanda $\mathrm{H}$, et al. Male involvement in PMTCT services in Mbeya region, Tanzania. AIDS Behav 2009;13 Suppl 1:92-102.

9 Torpey K, Kabaso M, Kasonde P, et al. Increasing the uptake of prevention of mother-to-child transmission of HIV services in a resource-limited setting. BMC Health Serv Res 2010;10.

10 Gourlay A, Birdthistle I, Mburu G, et al. Barriers and facilitating factors to the uptake of antiretroviral drugs for prevention of motherto-child transmission of HIV in sub-Saharan Africa: a systematic review. J Int AIDS Soc 2013;16:18588.

11 Montgomery E, van der Straten A, Torjesen K. "Male involvement" in women and children's HIV prevention: challenges in definition and interpretation. J Acquir Immune Defic Syndr 2011;57:e114-6.

12 Galle A, Cossa H, Griffin S, et al. Policymaker, health provider and community perspectives on male involvement during pregnancy in southern Mozambique: a qualitative study. BMC Pregnancy Childbirth 2019;19:384-30. doi:10.1186/s12884-019-2530-1

13 Comrie-Thomson L, Tokhi M, Ampt F, et al. Challenging gender inequity through male involvement in maternal and newborn health: critical assessment of an emerging evidence base. Cult Health Sex 2015;17(Suppl 2):177-89. doi:10.1080/13691058.2015.1053412

14 Brubaker K, Nelson BD, McPherson H, et al. Qualitative study of the role of men in maternal health in resource-limited communities in Western Kenya. Int J Gynaecol Obstet 2016;135:245-9.

15 Peneza AK, Maluka SO. 'Unless you come with your partner you will be sent back home': strategies used to promote male involvement in antenatal care in southern Tanzania. Glob Health Action 2018;11:1449724.

16 Dumbaugh M, Tawiah-Agyemang C, Manu A, et al. Perceptions of, attitudes towards and barriers to male involvement in newborn care in rural Ghana, West Africa: a qualitative analysis. BMC Pregnancy Childbirth 2014;14:269.

17 Matseke MG, Ruiter RAC, Barylski N, et al. A qualitative exploration of the meaning and understanding of male partner involvement in pregnancy-related care among men in rural South Africa. J Soc Behav Health Sci 2017;11.

18 Bawadi HA, Qandil AM, Al-Hamdan ZM, et al. The role of fathers during pregnancy: A qualitative exploration of Arabic fathers' beliefs. Midwifery 2016;32:75-80.

19 Maman S, Moodley D, Groves AK. Defining male support during and after pregnancy from the perspective of HIV-Positive and HIV-Negative women in Durban, South Africa. J Midwifery Womens Health 2011;56:325-31. doi:10.1111/j.1542-2011.2011.00029.x

20 Greenhill E, Vollmer RL. Perceptions of a Father's Role during a Couple's First Pregnancy. Fam Consum Sci Res J 2019;48:52-64.

21 Alio AP, Lewis CA, Scarborough $\mathrm{K}$, et al. A community perspective on the role of fathers during pregnancy: a qualitative study. $B M C$ Pregnancy Childbirth 2013;13:60.

22 Ampt F, Mon MM, Than KK, et al. Correlates of male involvement in maternal and newborn health: a cross-sectional study of men in a peri-urban region of Myanmar. BMC Pregnancy Childbirth 2015;15.

23 Byamugisha R, Tumwine JK, Semiyaga N, et al. Determinants of male involvement in the prevention of mother-to-child transmission of HIV programme in eastern Uganda: a cross-sectional survey. Reprod Health 2010;7:12.

24 Galle A, De Melo M, Griffin S, et al. A cross-sectional study of the role of men and the knowledge of danger signs during pregnancy in southern Mozambique. BMC Pregnancy Childbirth 2020;20:572.

25 Lamb ME. The history of research on father involvement. Marriage Fam Rev 2000;29:23-42.

26 Silge J, Robinson D. tidytext: text mining and analysis using Tidy data principles in R. J Open Source Softw 2016;1:37.

27 Topic modeling visualization - How to present results of LDA model? | ML+ [Internet]. Available: https://www.machinelearningplus.com/ nlp/topic-modeling-visualization-how-to-present-results-Ida-models/

28 Pop VJM, Pommer AM, Pop-Purceleanu M, et al. Development of the Tilburg pregnancy distress scale: the TPDS. BMC Pregnancy Childbirth 2011:11:80

29 Rini C, Schetter CD, Hobel CJ, et al. Effective social support: antecedents and consequences of partner support during pregnancy. Pers Relatsh 2006;13:207-29.

30 Suandi D, Williams $P$, Bhattacharya S. Does involving male partners in antenatal care improve healthcare utilisation? Systematic review and meta-analysis of the published literature from low- and middleincome countries. Int Health 2020;12:484-98.

31 Lee JY, Knauer HA, Lee SJ, et al. Father-inclusive perinatal parent education programs: a systematic review. Pediatrics 2018;142:e20180437.

32 Kululanga LI, Sundby J, Malata A, et al. Striving to promote male involvement in maternal health care in rural and urban settings in Malawi - a qualitative study. Reprod Health 2011;8:36.

33 Påfs J, Musafili A, Binder-Finnema P, et al. 'They would never receive you without a husband': paradoxical barriers to antenatal care scaleup in Rwanda. Midwifery 2015;31:1149-56.

34 Påfs J, Musafili A, Binder-Finnema P, et al. 'They would never receive you without a husband': Paradoxical barriers to antenatal care scaleup in Rwanda. Midwifery 2015;31:1149-56.

35 Triulzi I, Palla I, Ciccacci F, et al. The effectiveness of interventions to involve men living with HIV positive pregnant women in low-income countries: a systematic review of the literature. BMC Health Serv Res 2019;19:943.

36 Audet CM, Chire YM, Vaz LME, et al. Barriers to male involvement in antenatal care in rural Mozambique. Qual Health Res 2016;26:1721-31.

37 Morgan R, Tetui M, Muhumuza Kananura R, et al. Gender dynamics affecting maternal health and health care access and use in Uganda. Health Policy Plan 2017;32:v13-21.

38 Taghizadeh Z, Ravaghi H, Sarafraz N. Designing a model for promoting the participation of men in sexual and reproductive health using structural equation modeling-partial least squares (Sem-pls). Electron J Gen Med 2019;16.

39 Sahu D, Dutta T, Kumar S, et al. Effects of Women's Autonomy and Male Involvement on Reproductive and Child Health $(\mathrm{RCH})$ Service Utilization in Uttar Pradesh. Open J Prev Med 2016;06:260-71.

40 August F, Pembe AB, Mpembeni R, et al. Community health workers can improve male involvement in maternal health: evidence from rural Tanzania. Glob Health Action 2016;9:30064

41 Women's Position within the Household as a Determinant of Maternal Health Care Use in Nepal on JSTOR [Internet]. Available: https://www.jstor.org/stable/4147608?casa token=wValf PXfJcAAAAA\%3AxJIJRgSjzWb54D0XkinKBPUnwFiOrmqcYk D8qqS_w82Pv8aUT8i-xNrIByOGtE09pR7T7GfXVz4m1ildPB5X nGLs2q1pIOYsFehfRICiCeGHBGCq4g\&seq=1\#metadata_info_tab_ contents

42 Jennings L, Na M, Cherewick M. Women's empowerment and male involvement in antenatal care: analyses of Demographic and Health Surveys (DHS) in selected African countries, 2014. Available: http:// www.biomedcentral.com/1471-2393/14/297

43 Ruane-McAteer E, Gillespie K, Amin A, et al. Gender-transformative programming with men and boys to improve sexual and reproductive health and rights: a systematic review of intervention studies. $B M J$ Glob Health 2020;5:e002997.

44 Turan JM, Nalbant $\mathrm{H}$, Bulut A, et al. Including expectant fathers in antenatal education programmes in Istanbul, Turkey. Reprod Health Matters 2001;9:114-25-25.

45 Kunene B, Beksinska M, Zondi S. Involving men in maternity care: South Africa [Internet]. Reproductive Health Social and Behavioral Science Research, 2004. Available: https://knowledgecommons. popcouncil.org/departments_sbsr-rh/457

46 Damra JK, Abujilban SK, Rock MP, et al. Pregnant Women's Experiences of Intimate Partner Violence and Seeking Help from Health Care Professionals: A Jordanian Qualitative Study. J Fam Violence 2015;30:807-16

47 Spangaro J, Koziol-McLain J, Zwi A, et al. Deciding to tell: qualitative configurational analysis of decisions to disclose experience of intimate partner violence in antenatal care. Soc Sci Med 2016;154:45-53.

48 Doyle K, Levtov RG, Barker G, et al. Gender-transformative Bandebereho couples' intervention to promote male engagement in reproductive and maternal health and violence prevention in Rwanda: Findings from a randomized controlled trial. PLoS One 2018;13:e0192756

49 Levy JK, Darmstadt GL, Ashby C, et al. Characteristics of successful programmes targeting gender inequality and restrictive gender norms for the health and wellbeing of children, adolescents, and young adults: a systematic review. Lancet Glob Health 2020;8:e225-36.

50 Baldwin S, Bick D. First-Time fathers' needs and experiences of transition to fatherhood in relation to their mental health and wellbeing: a qualitative systematic review protocol. JBI Database System Rev Implement Rep 2017;15:647-56.

51 Deave T, Johnson D, Ingram J. Transition to parenthood: the needs of parents in pregnancy and early parenthood. BMC Pregnancy Childbirth 2008;8:30. 
52 ICPD AND HUMAN RIGHTS: 20 years of advancing reproductive rights trough UN treaty bodies and legal reform [Internet], 2013. Available: www.reproductiverights.org

53 Lee JY, Knauer HA, Lee SJ, et al. Father-inclusive perinatal parent education programs: a systematic review. Pediatrics 2018;142. doi:10.1542/peds.2018-0437. [Epub ahead of print: 14 Jun 2018].

54 Onyeze-Joe C, Godin I. Experiences, views and needs of first-time fathers in pregnancy-related care: a qualitative study in south-east Nigeria. BMC Pregnancy Childbirth 2020;20:213. doi:10.1186/ s12884-020-02889-w

55 Sahu SK, Kumar SG, Bhat BV, et al. Malnutrition among underfive children in India and strategies for control. J Nat Sci Biol Med 2015;6:18-23.

56 Piot P, Legido-Quigley H. Global Perspectives on Human Immunodeficiency Virus Infection and Acquired Immunodeficiency Syndrome. In: Mandell, Douglas, and Bennett's Principles and Practice of Infectious Diseases, 2014.

57 Zegeye A, Alebel A, Gebrie A, et al. Prevalence and determinants of antenatal depression among pregnant women in Ethiopia: a systematic review and meta-analysis. BMC Pregnancy Childbirth 2018;18:462

58 Blaney NT, Fernandez MI, Ethier KA, et al. Psychosocial and behavioral correlates of depression among HIV-infected pregnant women. AIDS Patient Care STDS 2004;18:405-15.

59 Hartley M, Tomlinson M, Greco E, et al. Depressed mood in pregnancy: prevalence and correlates in two Cape town peri-urban settlements. Reprod Health 2011;8:9.

60 Barthel D, Kriston L, Barkmann C, et al. Longitudinal course of ante- and postpartum generalized anxiety symptoms and associated factors in West-African women from Ghana and Côte d'Ivoire. J Affect Disord 2016;197:125-33.

61 Woody CA, Ferrari AJ, Siskind DJ, et al. A systematic review and meta-regression of the prevalence and incidence of perinatal depression. J Affect Disord 2017;219:86-92.

62 Sowa NA, Cholera R, Pence BW, et al. Perinatal depression in HIV-infected African women: a systematic review. J Clin Psychiatry 2015;76:1385-96-96.

63 Ng'oma M, Bitew T, Kaiyo-Utete M, et al. Perinatal mental health around the world: priorities for research and service development in Africa. BJPsych Int 2020;17:56-9.

64 Gajaria A, Ravindran AV. Interventions for perinatal depression in low and middle-income countries: a systematic review. Asian J Psychiatr 2018;37:112-20.

65 Peltzer K, Rodriguez VJ, Lee TK, et al. Prevalence of prenatal and postpartum depression and associated factors among HIV-infected women in public primary care in rural South Africa: a longitudinal study. AIDS Care 2018;30:1372-9.

66 Page-Reeves J, Regino L, Murray-Krezan C, et al. A comparative effectiveness study of two culturally competent models of diabetes self-management programming for Latinos from low-income households. BMC Endocr Disord 2017;17:46.

67 Vanobberghen F, Weiss HA, Fuhr DC, et al. Effectiveness of the thinking healthy programme for perinatal depression delivered through Peers: pooled analysis of two randomized controlled trials in India and Pakistan. J Affect Disord 2020;265:660-8.

68 Ola BA, Atilola O. Task-shifted interventions for depression delivered by lay primary health-care workers in low-income and middleincome countries. Lancet Glob Health 2019;7:e829-30.

69 Pilkington PD, Whelan TA, Milne LC. A review of partner-inclusive interventions for preventing postnatal depression and anxiety. Clin Psychol 2015;19:63-75

70 Peltzer K, Abbamonte JM, Mandell LN, et al. The effect of male involvement and a prevention of mother-to-child transmission (PMTCT) intervention on depressive symptoms in perinatal HIVinfected rural South African women. Arch Womens Ment Health 2020;23:101-11.

71 Maselko J, Hagaman AK, Bates LM, et al. Father involvement in the first year of life: associations with maternal mental health and child development outcomes in rural Pakistan. Soc Sci Med 2019;237:112421.

72 Yeganeh N, Simon M, Mindry D, et al. Barriers and facilitators for men to attend prenatal care and obtain HIV voluntary counseling and testing in Brazil. PLoS One 2017;12:e0175505.

73 Yeganeh N, Kerin T, Simon M, et al. Challenges and motivators for male partner involvement in prenatal care for HIV testing in a tertiary setting in Brazil. Int J STD AIDS 2019;30:875-84.

74 Bonifácio LP, Franzon ACA, Zaratini FS, et al. PRENACEL partner use of short message service (SMS) to encourage male involvement in prenatal care: a cluster randomized trial. Reprod Health 2020;17:45-12.

75 Berti PR, Sohani S, Costa Eda, et al. An adequacy evaluation of a maternal health intervention in rural Honduras: the impact of engagement of men and empowerment of women. Rev Panam Salud Publica 2015;37:90-7.

76 Barker G, Verani F. A critical literature review with policy considerations

77 Santos SJ. MenCare in Latin America: Challenging Harmful Masculine Norms and Promoting Positive Changes in Men's Caregiving EMERGE Case Study. 5, 2015.

78 Levtov RG, Barker G, Contreras-Urbina M. Pathways to genderequitable men: findings from the International men and gender equality survey in eight countries. Men Masc 2014;17:467-501.

79 Bearak J, Popinchalk A, Ganatra B, et al. Unintended pregnancy and abortion by income, region, and the legal status of abortion: estimates from a comprehensive model for 1990-2019. Lancet Glob Health 2020;8:e1152-61.

80 Altshuler AL, Nguyen BT, Riley HEM, et al. Male partners' involvement in abortion care: a mixed-methods systematic review. Perspect Sex Reprod Health 2016;48:209-19.

81 Kane J, Lohan M, Kelly C. Adolescent men's attitudes and decision making in relation to pregnancy and pregnancy outcomes: an integrative review of the literature from 2010 to 2017. J Adolesc 2019;72:23-31.

82 Malterud K. Qualitative research: Standards, challenges, and guidelines.. Lancet 2001;358:483-8. 